\title{
Current applications of high-resolution mass spectrometry for the analysis of new psychoactive substances: a critical review
}

\author{
Daniel Pasin ${ }^{1}$, Adam Cawley ${ }^{2}$, Sergei Bidny ${ }^{3}$, Shanlin $\mathrm{Fu}^{1 *}$ \\ ${ }^{1}$ Centre for Forensic Science, University of Technology Sydney, Broadway NSW 2007, \\ Australia \\ ${ }^{2}$ Australian Racing Forensic Laboratory, Racing NSW, Sydney NSW 2000, Australia \\ ${ }^{3}$ Forensic Toxicology Laboratory, NSW Forensic and Analytical Science Service, Lidcombe, \\ NSW 2141, Australia
}

Keywords: new psychoactive substances, high-resolution mass spectrometry, non-targeted screening

*Author to whom correspondence should be addressed:

Shanlin Fu

University of Technology Sydney

15 Broadway, Sydney NSW 2007, Australia

Email : $\underline{\text { Shanlin.Fu@uts.edu.au }}$ 


\begin{abstract}
The proliferation of new psychoactive substances (NPS) in recent years has resulted in the development of numerous analytical methods for the detection and identification of known and unknown NPS derivatives. High-resolution mass spectrometry (HRMS) has been identified as the method of choice for broad screening of NPS in a wide range of analytical contexts due to its ability to measure accurate masses using data-independent acquisition (DIA) techniques. Additionally, it has shown promise for non-targeted screening strategies that have been developed in order to detect and identify novel analogues without the need for certified reference materials (CRMs) or comprehensive mass spectral libraries. This paper reviews the applications of HRMS for the analysis of NPS in forensic drug chemistry, clinical and forensic toxicology. It provides an overview of the sample preparation procedures in addition to data acquisition, instrumental analysis and data processing techniques. Furthermore, it will give an overview of the current state of non-targeted screening strategies with discussion on future directions and perspectives of this technique.
\end{abstract}




\section{Introduction}

New psychoactive substances (NPS), designer drugs and "legal highs" are terms used to describe emerging novel compounds that are designed to exert similar pharmacological effects as traditional recreational drugs but intended to circumvent legislative measures [1-5]. Ultimately, most analogues eventually become controlled due to their unknown toxicological and pharmacological effects []. A dynamic situation exists between law-makers and clandestine laboratory operators that has caused rapid proliferation of novel analogues in recent years and, as such, makes the detection of these new derivatives potentially demanding in forensic analyses in different contexts such as forensic drug chemistry, clinical toxicology and forensic toxicology. According to the latest European Drug Report, more than 500 NPS analogues were reported to the European Union (EU) Early Warning System (EWS) over the last decade with the number of new analogues reported in the last five years comprising approximately $80 \%$ of the total number [7]. The highest number of new analogues reported for the first time was in 2014 with 101 analogues ( 20\%). The NPS market has been traditionally dominated by synthetic cannabinoids (SCs) with 157 of the 467 analogues (34\%) reported between 2009 and 2015, however, psychedelic tryptamines, piperazines and hallucinogenic phenethylamines were predominant between 2005 and 2007. In addition to SCs, synthetic cathinones also dominate the NPS market with 93 analogues (20\%) reported between 2009 and 2015 and higher numbers of analogues reported compared to SCs in 2010 and $2015[\underline{7}, \underline{8}]$. More recently there has been an emergence of synthetic opioid and designer benzodiazepine derivatives which have exhibited much higher potencies than their traditional counterparts $[\underline{9}, \underline{10}]$. These trends, in addition to the lack of available certified reference materials (CRMs) for novel analogues, highlights the fact that traditional targeted screening techniques are inadequate in handling the rapid proliferation of NPS subsequently allowing them to potentially go undetected in routine screening analyses [11]. 
Traditionally, the analysis of NPS has been typically performed using conventional gas chromatography - mass spectrometry (GC-MS) or liquid chromatography - tandem mass spectrometry (LC-MS/MS) using triple quadrupole (QqQ) mass analyzers for screening and quantification of NPS. More recently, high-resolution mass spectrometry (HRMS) has grown in popularity due to its ability to measure accurate masses and operate in data-independent acquisition (DIA) modes [12]. This acquisition technique provides comprehensive full scan MS and MS/MS that can be retrospectively interrogated for new analytes of interest without the need for re-extraction and re-analysis in the application of non-targeted/untargeted screening.

This review will focus on the use of HRMS in the analysis of NPS, particularly highlighting the different techniques used for sample preparation, instrumental analysis, data acquisition and data processing in a range of matrices in different analytical contexts such as forensic drug chemistry and clinical and forensic toxicology. Since there is scope for HRMS to be utilized for non-targeted screening, an overview of the current applications of this technique will be provided with discussion on its potential future directions.

\section{Analysis of NPS using HRMS}

A literature search was performed using public domain repositories such as PubMed and ScienceDirect with the search term "new psychoactive" in combination with one or more of the following: "high-resolution", "mass spectrometry", "time-of-flight" and "orbitrap". A total of 95 articles published between 2010 and early 2017 with 68 original research articles related to the analysis of NPS using HRMS were selected for inclusion in this review. The first publication describing the use of HRMS for the analysis of NPS was in 2010 regarding the metabolism of 3,4-methylenedioxypyrovalerone (MPDV) published by Meyer et al. [13] and was followed by an increasing number of publications each year with a total of 25 
publications identified in 2016, double the number published in 2015 (Figure S1 in the Electonic Supplementary Material). The increasing use of HRMS has been highlighted by several reviews, mostly focusing on the role of this technique in forensic and clinical toxicology [14-16]. Recently, Maurer et al. [17] reviewed the use of HRMS in toxicology and attributed its increased popularity to factors such as the ability to differentiate compounds with identical nominal masses but different accurate masses and the ease at which new compounds can be added to already existing screening procedures. Furthermore, they highlighted limitations such as instrument cost, the complexity of data processing software and the requirement of skilled operators. Finally, Meyer et al. [15] reviewed literature pertaining to the use of LC coupled to low- and high-resolution instrumentation for screening of NPS in biological matrices. They concluded that LRMS is still the standard technique for quantitative analysis due to ease of operation and reasonable cost, however, HRMS is likely to become the gold standard for non-targeted screening in coming years, provided that instrument prices are reduced and data mining software becomes more user-friendly.

\section{Overview of the role HRMS in different analytical contexts}

HRMS is a versatile analytical technique that can be applied in different configurations with interchangeable ionization sources and sophisticated data acquisition capabilities. Applications may range from the generation of molecular formulae from accurate masses to the development of screening and quantitative methods and further to non-targeted screening approaches. Consequently, HRMS has become an important technique in drug testing for therapeutic, medico-legal and law enforcement purposes.

The analysis of seized materials in forensic drug chemistry is performed in accordance with the recommendations outlined by Scientific Working Group for the Analysis of Seized Drugs (SWGDRUG) [18]. Traditionally, this includes a combination of conventional analytical 
techniques such as GC-MS, Fourier-transform infrared (FTIR) spectroscopy and nuclear magnetic resonance (NMR) spectroscopy for confirmation and structural elucidation [11]. Recently, HRMS has been employed to confirm the proposed identity from GC-MS library matches by generating molecular formulae from acquired accurate masses. Unfortunately, in most cases involving NPS, commercial GC-MS libraries do not contain novel analogues. In this case, HRMS is often used to perform tandem MS (MS/MS) to evaluate the collisioninduced dissociation (CID) pathways for putative structural elucidation followed by confirmation using NMR spectroscopy. The aforementioned approaches have also been adopted for the analysis of purchased materials from "headshops" or from online vendors to determine the composition of "legal high" products available on the market. In both cases, the objective of these analyses is focused on the identification of active components and is qualitative rather than quantitative, however, determination of the purity of the material may be a requirement and can be determined by GC-MS or quantitative NMR.

In analytical toxicology, systematic toxicological analysis (STA) provides the identity and, in most circumstances, the concentrations of compounds present in biological matrices. For clinical toxicology, these analyses are typically qualitative and are intended to provide clinicians with information that is used to develop appropriate treatment plans for patients suspected of being intoxicated by particular substances. Ojanperä et al. [16] reviewed the role of HRMS and stated that emergency clinical toxicological analyses are often restricted to two hours for sample transportation, analysis and reporting and subsequently limiting the analytical techniques that can be used. Simultaneously, Wu et al. [19] highlighted that unknown compounds pose serious challenges for clinicians regarding the treatment of patients. In addition, they also indicated that traditional targeted methods are limited in their ability to detect and identify novel analogues and suggested that HRMS may be a solution to this problem by providing a comprehensive screening tool. 
For forensic toxicology, the same principals apply as clinical toxicology, however, the focus is shifted towards the requirements for results to be admissible and justifiable in a court of law [16]. Unlike clinical toxicology, forensic toxicology samples are typically subjected to a confirmation step following screening procedures and require fully validated quantitative procedures. Consequently, the detection and putative identification of novel analogues using non-targeted strategies bears no evidential weight since identification is limited by the requirement for the use of CRMs or mass spectral libraries.

Finally, the use of HRMS in metabolism studies has increased in recent years particularly due to the sophisticated software tools available [14]. Peters et al. [20] reviewed the recent developments of LC-MS for metabolism studies of NPS identifying that the main advantage of HRMS is the elimination of interferences that have the same nominal mass but different accurate masses. However, HRMS may not necessarily contribute more value to structural assignments of metabolites with intact product ions. The toxicokinetics of NPS have been comprehensively reviewed by Meyer []] and Ellefsen et al. [21] .

The role and application of HRMS is variable in different contexts, however, the general workflow is typically the same, which includes: sample preparation, instrumental analysis, data acquisition and data processing.

\section{Sample Preparation}

Sample preparation procedures are often a requirement prior to instrumental analysis to extract analytes of interest out of complex matrices to provide cleaner extracts for the purpose of maintaining instrument capability. The sample preparation techniques reported have varied between matrix and within matrix type depending on the level of sensitivity and specificity required for particular assays. 


\section{Seized materials and purchased legal highs}

These materials often exist in a wide variety of forms including powders or crystalline solids, herbal materials and blotter papers. Powders and crystalline solids were largely prepared using solvent extraction or dilution typically with methanol or acetonitrile for MS analyses utilizing LC or GC separation. Schevyrin et al. [22-24] dissolved smoke mixtures in methanol followed by an additional filtration step with a cellulose membrane. The extraction of blotter papers has been achieved by soaking the paper in methanol for 6 hours [25-27].

\section{Biological matrices}

For blood, sample preparation procedures included protein precipitation, liquid-liquid extraction (LLE), salting-out assisted liquid-liquid extraction (SALLE) and microwaveassisted extraction (MAE) [28-32]. Glicksberg et al. [28] reported the use of protein precipitation with acetonitrile followed by solid-phase extraction (SPE) for the extraction of synthetic cathinones from blood plasma and urine. Extraction efficiencies of greater than $80 \%$ and $90 \%$ were reported for plasma and urine, respectively. The use of acetonitrile as a solvent for LLE [30] and SALLE [31] procedures has shown promise for the extraction of synthetic cathinones and synthetic cannabinoids with average recoveries greater than $80 \%$.

The analysis of NPS in urine has typically been limited to the detection of parent molecules after extraction by SPE or LLE without the use of enzymatic or acid hydrolysis. Concheiro et al. [33, 34] utilized SPE for the extraction of synthetic cathinones and other NPS from 100 $\mu \mathrm{L}$ of urine using strong cation exchange (SCX) cartridges. They observed recoveries for most analytes to be greater than 90\%. Archer et al. [35-37] has reported the use of LLE and SPE for the analysis of urine samples obtained from street urinals. In addition, SPE has been commonly used for the extraction of wastewater samples for epidemiological studies [38-40]. Bäckberg et al. [41] reported the use of a dilute and shoot method for the detection of 
MDMB-CHMICA in the urine of patients with non-fatal intoxications. However, the parent molecule was not detected in urine but could be quantified in serum. Enzymatic hydrolysis with $\beta$-glucuronidase has been performed in a limited number of cases prior to SPE. [느, $\underline{43}]$. Sundström et al. [43] developed a NPS screening method in urine which included various hydroxylated and carboxylated synthetic cannabinoids metabolites which were positively detected in authentic urine samples.

The analysis of hair has been investigated in a limited number of studies with sample preparation procedures including MAE [32] and solvent extraction following ball mill pulverization [44]. Montesano et al. [45] evaluated the use of pressurized liquid extraction (PLE) for the extraction of various NPS analogues from cut hair following external decontamination. They investigated the use of different extraction temperatures and solvents and found that recoveries were typically higher with water and methanol $(90: 10 \mathrm{v} / \mathrm{v})$ at $125^{\circ} \mathrm{C}$, however, potential thermo-degradation should be considered when extracting at such high temperatures.

\section{Instrumental Analysis Techniques}

Over the years, many instrumental techniques have been investigated and evaluated for the analysis of NPS in different matrices in a variety of contexts. In most cases, conventional chromatographic methods such as reversed-phase LC and GC are commonly used particularly in toxicological assays where chromatographic separation is often necessary for complex biological matrices. Additionally, the use of direct sampling techniques has also been reported, particularly in forensic drug chemistry. Figure S2 summarizes the frequency of chromatographic and direct sampling techniques. These techniques are typically coupled or interfaced with hybrid quadrupole time-of-flight (QTOF) mass spectrometers. However, the use of TOF instruments, high-resolution ion trap instruments such as the Thermo Fisher 
Scientific (Bremen, Germany) hybrid quadrupole-Orbitrap (Q Exactive ${ }^{\mathrm{TM}}$ ) and hybrid linear ion trap-Orbitrap (LTQ Orbitrap XL ${ }^{\mathrm{TM}}$ ) mass analyzers are also common. Figure S3 summarizes the frequency of different HRMS platforms.

\section{Conventional separation techniques coupled to HRMS}

LC has been the most commonly adopted conventional separation technique coupled to HRMS for the analysis of NPS due to the well-recognized fact that analytes of interest do not need to be volatile and do not require derivatization $[\underline{15}, \underline{28}, \underline{30}, \underline{33}, \underline{39}, \underline{42}, \underline{43}, \underline{46-48}$. Subsequently, the use of GC coupled to HRMS for the analysis of NPS has been limited. Ojanpera et al. [49] reported the use of GC-QTOF-MS with atmospheric pressure chemical ionization (APCI) for the detection and quantification of a small of number of NPS in sheep blood without derivatization. Few studies have involved the use of both LC and GC coupled to HRMS. Schevyrin et al. [22-24] published a series of articles reporting the detection of several SCs for the first time in smoke mixtures from Russia and Belarus using both LC and GC coupled to QTOF-MS. The combination of both chromatography methods was used in lieu of these compounds having spectra in commercial libraries for GC-MS. Furthermore, the use of capillary electrophoresis (CE) coupled to QTOF-MS has been only reported once for the analysis of NPS in serum and hair by Woźniakiewicz et al. [32] with its limited use likely due to inconsistent migration times and reduced sensitivity compared to conventional LC and GC [50].

\section{Direct sample analysis techniques coupled to HRMS}

Recently, there has been a significant growth in the use of direct sampling analysis techniques, particularly in the analysis of seized materials. The advantage of this technique is the ability to rapidly analyze samples without chromatographic separation and with minimal to no sample preparation. Direct sampling techniques have been performed using an LC 
system where the sample is injected into the carrier flow that is delivered directly into the MS bypassing the chromatographic column. This technique is known as flow injection analysis (FIA) and has been reported on a single occasion by Alechaga et al. [51] for rapid, widerange screening of NPS in "legal highs". Although this technique is rapid, it still requires minimal sample preparation such as dissolution of the sample in an appropriate solvent.

In addition, the use of matrix-assisted laser desorption ionization (MALDI) and desorption electrospray ionization (DESI) coupled to HRMS has been reported for the analysis of NPS [52-54]. Ostermann et al. [52] analyzed NPS CRMs and authentic samples using MALDI coupled to an LTQ-Orbitrap. It was indicated that sample preparation and analysis (MS and MS/MS) could be performed in approximately four minutes providing a rapid analytical technique. MALDI offers relatively simplistic sample preparation and easy-to-interpret mass spectra, however, considerations need to be made regarding matrix selection and laser power to generate optimal results. More novel techniques such as proton transfer reaction (PTR) [55] and selective reagent ionization (SRI) [56, 57] coupled to QTOF-MS have been reported, however, their use has been limited.

The most commonly adopted ambient ionization technique is direct analysis in real-time (DART) mass spectrometry which is capable of rapidly analyzing samples without sample preparation. Briefly, DART-MS consists of two orifices (the outlet of the ion source and the inlet of the MS), samples are placed in between these orifices and their molecules interact with metastable ions created by the ion source which in turn produce monoisotopic ions $[\mathrm{M}+\mathrm{H}]^{+}$or $[\mathrm{M}-\mathrm{H}]^{-}$for basic and acidic molecules, respectively [58]. DART-MS has traditionally been coupled to TOF instruments particularly the JEOL AccuTOFTM DART (Tokyo, Japan), however, they have also been coupled with QTOF and Orbitrap mass analyzers. While this technique has been largely adopted for the analysis of seized materials, it has not found applications in analytical toxicology. 
Poklis et al. [59] were the first to publish work that utilized DART coupled to HRMS for the analysis of purchased legal highs using an AccuTOFTM DART. They analyzed two products purchased on the internet, "Raving Dragon Novelty Bath Salts" and "Raving Dragon Voodoo Dust", and found them to contain methylone and pentedrone, respectively. This study also highlighted the adaptive nature of the drug market and how it responds to legislative changes. The first product, purchased in February 2011 was found to contain methylone which was uncontrolled at the time. However, this product was removed from the market in October of the same year due to subsequent scheduling of mephedrone, methylone and MDPV and replaced with the second product in February 2012. This product was found to contain pentedrone which was not scheduled under the Controlled Substances Act (United States).

Musah et al. [60] reported the use of an AccuTOFTM DART with in-source CID to analyze pure synthetic cathinone standards or mixtures of cathinones with adulterants/cutting agents without sample preparation. Precursor ions for all cathinones and adulterants were detected and components of the mixtures could be identified by looking at the individual pure cathinone MS/MS spectra for each component. In addition, mixtures containing multiple cathinones could also be differentiated. They highlighted that due to the rapid analysis time samples containing synthetic cathinones and cutting agents could be triaged and this could be a viable option for reducing routine casework backlogs and provide the necessary information to make decisions on further confirmatory testing methods.

DART-MS has been also investigated for the analysis of SCs in seized herbal materials. Habala et al. [58] analyzed 8 samples using a DART source coupled to an LTQ Orbitrap $\mathrm{XL}^{\mathrm{TM}}$ mass analyzer. The samples were analyzed using different methods including direct analysis of the solid herbal material and methanolic extracts. They positively identified six synthetic cannabinoids in the seized samples with identification achieved by comparing the MS/MS data with those reported in literature in addition to GC-MS data compared with the 
SWGDRUG library. However, a drawback of directly analyzing herbal material is often the inhomogeneity of the samples and it is recommended that sampling is conducted in replicate and at different locations within the seized exhibit. It was also discovered that the leaves have a greater concentration than the stems of plant material. This study highlights the viability of DART-MS for the analysis of herbal materials that does not rely on solvent selection and chromatographic methods for separation.

Comprehensive screening of NPS using DART-MS has also been reported by Gwak et al. [61] who developed a method to screen for 35 NPS using DART coupled to a hybrid QTOFMS. The panel comprised of mostly synthetic cathinones and SCs with a single phenethylamine derivative (25I-NBOMe) and all analytes were detected with mass errors within $\pm 5 \mathrm{ppm}$. However, the panel of selected analytes contained many isomeric compounds that could not be differentiated by full scan MS since there is no separation component in DART-QTOF-MS. They also highlighted that the use of a QTOF mass analyzer instead of the most commonly used AccuTOFTM provides the ability to simultaneously collect full scan MS and MS/MS data. They also compared the product ion spectra of the selected analytes generated using DART and ESI and found that the relative intensities were similar to those provided by ESI, allowing for comparison of DART-MS data with spectral libraries developed using ESI. In addition, they also assessed the limit of detection for the DART-QTOF-MS and found that all analytes had LODs between 300 to 340 pg. This screening method was successfully applied to authentic samples with the identification based on comparison with spectral libraries, however, they could not detect both compounds in one of the samples due to the data-dependent acquisition (QTOFF). It was stated that the "Auto MS/MS" function selects only the most abundant peak for CID, however, to our knowledge, multiple precursors can be selected using this function that would allow MS/MS spectra to be produced for both analytes. 
Although this technique is rapid and high-throughput, there are certain limitations that should be considered. Firstly, since there is no chromatographic separation, isobaric compounds cannot be differentiated if their precursor and product ions are identical such as the positional isomers of 4-methylmethcathinone (mephedrone). Secondly, complex spectra containing analytes of interest, carrier flow components and interferences are produced due to the fact that all ions are recorded within the very short time frame that can be difficult to interpret particularly for MS/MS.

\section{Data Acquisition}

HRMS offers powerful data acquisition techniques compared to those employed by lowresolution mass analyzers and has the capability of being operated in a number of acquisition modes. The simplest data acquisition technique, which is a generic feature for all HRMS platforms, is full scan MS mode which measures all intact masses that reach the detector. This provides accurate $\mathrm{m} / \mathrm{z}$ values that can be used to generate chemical formulae with high mass accuracy $(<5$ ppm mass error). Archer et al. [37] employed only full scan MS for the analysis of NPS in pooled urine samples indicating that if there was the possible presence of a substance that the residue could be re-analysed using tandem MS (MS/MS) or MS/MS/MS $\left(\mathrm{MS}^{3}\right)$. However, it is unclear as to why they used only full scan MS considering they were using a LTQ Orbitrap XL ${ }^{\mathrm{TM}}$ which is capable of collecting MS and MS/MS data simultaneously, removing the need for re-analysis. Determination of chemical formulae and double bond equivalents (or degree of unsaturation; DBE) based on accurate mass can provide important information, however, it does not provide information on chemical structure, therefore, MS/MS is used for CID to provide putative structural elucidation.

MS/MS can be either tandem-in-space such as hybrid-quadrupole time-of-flight (QTOF) mass analyzers or tandem-in-time such as linear ion Orbitrap instruments. In addition, MS/MS can be operated in either DDA or DIA modes. 


\section{Data-dependent MS/MS acquisition}

DDA operates by taking an initial MS survey scan and then MS/MS events are triggered if precursor ions in the preceding scan meet predefined criteria such as an intensity threshold for $n$-selected precursor ions. This acquisition mode can be operated in an "untargeted" manner where MS/MS events are triggered based on the abundance of precursor ions in the preceding MS scan. A limitation of this technique is that it is possible for MS/MS events to be triggered by abundant and irrelevant compounds such as background or contamination ions that may necessitate reanalysis if analytes of interest did not trigger an MS/MS event. Andrés-Costa et al.[38] reported the use of information-dependent acquisition (IDA) for the quantification of NPS in wastewater using a Sciex TripleTOF (Framingham, MA, USA). Precursor ions that exceeded 1000 counts and were within an ion tolerance of $10 \mathrm{mDa}$ were selected for MS/MS at a collision energy of $40 \mathrm{eV}$. They suggested that data can be acquired for multiple CEs in separate injections for lower intensity precursor ions or by adding IDA functions at different collision energies for higher intensity ions. The lowest calibration level for each analyte was determined by the concentration that gave an intensity $\geq 10,000$ counts to ensure that MS/MS was triggered for lower concentrations and with achievable identification. To increase the coverage of precursor ions subjected to MS/MS it may be reasoned that the number of MS/MS events per scan can simply be increased, however, the cycle time (MS scan $+n$ MS/MS scans) should be considered in conjunction with chromatographic peak widths.

Additionally, DDA can be operated in a targeted precursor selection mode whereby MS/MS events are triggered when pre-selected mass-to-charge $(\mathrm{m} / \mathrm{z})$ ratios in inclusions lists are detected in the preceding MS scan [28, $\underline{45}, \underline{49}]$. These inclusion lists are typically populated with precursor ion data and, if known, retention times obtained from CRMs. Inclusion of retention time for a particular $\mathrm{m} / \mathrm{z}$ value will only trigger MS/MS events for a specified 
retention time window. Furthermore, masses can be specified for MS/MS exclusion, such as common background and interference ions. Concheiro et al. $[\underline{33}, \underline{34}]$ reported the use of datadependent MS/MS (ddMS ${ }^{2}$ ) with an inclusion list using a Q Exactive ${ }^{\mathrm{TM}}$. Detected precursor ions above the optimized threshold (up to 10 per cycle) were filtered by the quadrupole, fragmented by higher energy collision dissociation (HCD) followed by collection in the Ctrap and analysed by the Orbitrap. Targeted DDA ensures that only analytes of interest trigger MS/MS events making the data analysis procedure significantly easier. However, a limitation of this acquisition technique is that it does not allow for retrospective data interrogation of new compounds if they were not subjected to MS/MS, therefore, samples would need to be extracted and reanalyzed which may not be possible if they are no longer available.

Fortunately, complementary targeted and "untargeted" DDA can be performed in a single analysis. This technique operates by conducting an MS scan followed by targeted MS/MS using an inclusion list and then "untargeted" MS/MS on $n$-selected precursors. Gonzálezmariño et al. [40] reported the use of a DDA acquisition mode for screening of NPS that involved MS/MS events for the top 5 most abundant precursor ions in the MS scan. Additionally, the DDA method also had an inclusion list of precursor ions for analytes that did not have reference standards available. They also performed targeted screening using MS data from SC and cathinone reference standards.

More recently in 2016, Qian et al. [62] detected and identified four new SCs (ADBBINACA, AB-FUBICA, ADB-FUBICA and AB-BICA) in a seized sample from a dismantled clandestine laboratory in China. DDA acquisition was performed with an MS scan succeeded by an MS/MS scan with a sweeping collision energy ( $25 \pm 15 \mathrm{~V}$ in this case $)$ to generate product ion spectra.

\section{Data-independent MS/MS acquisition}


DIA techniques subject all precursor ions detected in the MS scan to CID, providing full scan MS/MS. DIA is typically achieved by the rapid alternation between low and high energy channels. Most vendors offer DIA techniques such as $\mathrm{MS}^{\mathrm{E}}$ (Waters Corporation, Milford, MA, USA), All Ions MS/MS (Agilent Technologies, Santa Clara, CA, USA), broadband CID (bbCID, Bruker, Billerica, MA, USA). More novel DIA techniques such as sequential window acquisition of all theoretical mass spectra (SWATH®, Sciex) have been developed which involves sequential CID of mass range increments. Kinyua et al. [ $\underline{63}, \underline{64}]$ reported the use of All Ion MS/MS in two separate studies for qualitative screening of NPS in biological matrices. The data was acquired using three scan segments with different collision energies $(0,15$ and $35 \mathrm{eV})$ to provide the precursor ion $(0 \mathrm{eV})$ and product ions at low and high collision energies (15 and $35 \mathrm{eV}$, respectively). Sundström et al. [43] developed and validated a broad screening and quantification method for SCs and cathinones using bbCID, with reliable identification when compared to a database of 277 compounds

Baz-Lomba et al. [39] conducted screening of psychoactive substances including a limited number of NPS in wastewater samples using $\mathrm{MS}^{\mathrm{E}}$. A MS scan was acquired using a collision energy of $6 \mathrm{eV}$ in the first low energy function followed by a collision energy ramp from 15 to $50 \mathrm{eV}$ in the second high energy function. Similarly, Pasin et al. [31] analyzed 37 NPS (mostly cathinones) in post-mortem blood using a first function collision energy of $6 \mathrm{eV}$ and a second function collision energy ramp of $10-40 \mathrm{eV}$ to produce fragment-rich product ion spectra. The major advantage of DIA techniques is that it offers full retrospective data interrogation capabilities with the acquisition of full scan data for both MS and MS/MS [44]. The main limitation of DIA is that due to multiple precursor ions simultaneously subjected to $\mathrm{CID}$ in the same MS/MS event, spectra containing product ions for all precursor ions are generated ('chimeric spectra'). These spectra can be difficult to interpret if the product ions cannot be associated with the correct precursor ion [ㄷ]]. 
An advantage of tandem-in-time MS/MS techniques is that higher order or multi-stage MS/MS experiments $\left(\mathrm{MS}^{n}\right)$ can be performed. This involves an initial CID experiment on the precursor ion followed by a subsequent CID on a selected product ion. This is possible in tandem-in-time instruments because trapped ions are subjected to a collision energy and selectively ejected with the process repeated to the $n^{\text {th }}$ degree. $\mathrm{MS}^{2}$ is the most commonly used acquisition technique in routine analyses, however, $\mathrm{MS}^{n}$ is often used for elucidation of product ions. While there have been no $\mathrm{MS}^{n}$ studies reported using ion-trap instruments, "pseudo-MS" ${ }^{3}$ " experiments using QTOF instruments for structural elucidation have been performed by applying high source voltages to induce in-source CID followed by CID using the collision cell $[\underline{25-27}, \underline{66}, \underline{67}]$. This technique was applied to the structural elucidation of new SC derivatives, AM(N)-2201, BIM-018 and BIM-2201. Pseudo-MS ${ }^{3}$ experiments were used to investigate the CID pathway of the common product ion $\mathrm{m} / \mathrm{z} 233.1085$ for both $\operatorname{AM}(\mathrm{N})-2201$ and BIM-2201 by using a fragmentor voltage of $260 \mathrm{~V}$ to induce in-source CID followed by targeted MS/MS [23].

\section{Data Processing Techniques}

Data processing techniques used for the detection and identification of analytes can vary from instrument-to-instrument due to the independent nature of software supplied by instrument vendors. In addition, the objective of an analysis can dictate what workflow is utilized in particular contexts (i.e. targeted vs. non-targeted). However, these techniques can be generally categorized into known and unknown data processing techniques (Figure 1). Known data processing techniques can be achieved through the use of CRMs (targeted screening) and compound libraries (suspect screening). Non-targeted screening will be discussed as a separate section. 


\section{Targeted screening}

Targeted screening strategies involve the automated interrogation of acquired sample data with data collected from CRMs such as retention time, precursor and product ion $\mathrm{m} / \mathrm{z}$ and can be used for analyte confirmation and quantification [ $[63, \underline{68}]$. Targeted screening methods have been commonly developed in analytical toxicology for the analysis of biological matrices such as blood $[\underline{30}, \underline{31]}$, urine $[\underline{33}, \underline{34}, \underline{47}, \underline{64]}$ or a combination of both [28]. For qualitative screening, methods can be updated easily with new CRMs, however, a limitation of developing broad in-house libraries that encompass a large number of candidate analytes is the cost of purchasing all necessary CRMs. In addition, the suppliers of CRMs may be located in overseas countries which can result in delays due to permit authorizations and shipping times by which time the analytes may have declined in popularity and disappeared off the market. Furthermore, since many of these compounds are becoming controlled they may be embargoed by enforcement agencies such as the US Drug Enforcement Administration (DEA) and require additional fees for purchase.

\section{Suspect screening}

An alternative approach to developing targeted screening methods is the use of suspect screening which involves the interrogation of acquired sample data using "suspect" compound-specific information in the absence of appropriate CRMs [69]. For a suspected compound, the chemical formula is known and, therefore, the accurate monoisotopic mass $\left([\mathrm{M}+\mathrm{H}]^{+} /[\mathrm{M}-\mathrm{H}]^{-}\right)$can be determined and in turn extracted [63]. Putative identification using suspect screening can then be confirmed by acquiring the appropriate CRM, if available. For the analysis of NPS, most suspect screening methods reported have involved the comparison of acquired sample data with mass spectral libraries for detection and identification. These libraries are often comprehensive, providing better coverage of NPS analogues than 
conventional targeted methods. Libraries can be typically obtained from instrument vendors or other third party commercial library developers that are curated and regularly updated for new compounds. Suspect screening has been used in isolation and as a complementary technique with targeted screening for greater analyte coverage [40]. Heikman et al. [70] reported the use of complementary targeted and suspect screening with an in-house database containing 500 compounds, CRMs were available for 280 of these compounds with the remainder comprising of rare NPS and their known and predicted metabolites. Some studies have stated that databases or libraries have been used to process data but provided no information on which databases were used or how many compounds were in these databases. The use of the SWGDRUG database has been reported on two occasions for the analysis of synthetic cannabinoids, however, this database is for LRMS EI-MS data only [ $\underline{58}, \underline{71]}$. Other commercial libraries such as the NIST/EPA/NIH NIST 08 and the department of Forensic Science of the Commonwealth of Virginia 2012 custom Druglist have been reported [59]. Custom databases populated with data from different sources have been developed and utilized in two studies [1, $\underline{72}]$, however, most databases described encompass many categories of drugs and not just NPS so it is unclear how comprehensive they actually are or the number of NPS derivatives is simply not reported.

Ford et al. [73] screened almost 100 samples including herbal blends, cigarettes, liquids and powders/pills obtained over a year period (2014-2015) in the United Kingdom using two different mass spectral libraries. The samples were analyzed using the data-independent $\mathrm{MS}^{\mathrm{E}}$ acquisition mode and compared to a SC mass spectral library with over 100 first, second and third generation derivatives. In addition, the acquired mass spectra were also compared to a comprehensive general screening that contains over 1300 compounds including approximately $10 \%$ NPS. Identification was based on mass accuracy $( \pm 5 \mathrm{ppm})$ of the precursor and product (qualifier) ion, an average isotopic match within $20 \%$ compared to the 
theoretical ratio and retention time within $\pm 0.3 \mathrm{~min}$. The most comprehensive database developed to date was reported by Kinyua et al. [63] who built an in-house library using the personal compound database and library (PCDL) manager with over 1500 entries using information from literature including from the European Monitoring Centre for Drugs and Drug Addiction (EMCDDA) and United Nations Office of Drugs and Crime (UNODC). Development of libraries typically relies on primary source information such as those acquired from CRMs and secondary sources such as information published in literature, thus, limiting databases to known compounds. In addition, commercial libraries are often updated with new analogues, however, it is unrealistic to believe that libraries are updated as soon as novel analogues are detected and therefore there will be a delay in detection capabilities.

\section{Non-targeted/untargeted screening strategies}

The recent proliferation of NPS has initiated considerable interest into the development of socalled "non-targeted" or "untargeted" screening strategies in order to detect and putatively identify novel compounds without the use of CRMs or mass spectral libraries. However, the use of the term "non-targeted screening" or "non-targeted analysis" has adopted different definitions over the years with an obvious lack of consistency in the use of this concept. Oberacher et al. [65] reviewed the use of non-targeted LC-MS strategies in forensic toxicology indicating that non-targeted analyses can be achieved by applying DDA or DIA techniques and comparing the collected data with mass spectral libraries. In addition, Lung et al. [72] reported the use of non-targeted analysis for the detection of NPS in clinical toxicology with DIA and suspect screening. The efficacy of non-targeted screening, in part, relies on the adoption of appropriate data acquisition techniques, however, data acquisition constitutes only one part of the non-targeted screening workflow [74]. The use of mass spectral libraries for identification of compounds has been advantageous previously, however, with the rapid proliferation of NPS analogues these libraries can no longer be relied 
upon for detection of novel analogues. This is due to the fact that there can be a considerable delay between the time a novel analogue has been identified and its subsequent entry into a mass spectral library. Therefore, to keep up with the rapidly changing NPS market, more innovative workflows need to be developed and implemented for the detection and identification of NPS.

Non-targeted analysis has been more accurately defined as the detection and identification of compounds in a sample in the absence of prior information or when the molecular content of a sample is unknown [75]. This has been widely used in food and environmental analysis [68, 76]. More prominently, however, non-targeted screening has been used in metabolomics for the detection of biomarkers present in samples after treatment with particular stimuli and the strategies developed have been used as a model or have been adopted for non-targeted analyses in other areas. Metabolomics-based non-targeted strategies are typically designed to identify all compounds in treatment samples and compare them to the control samples to observe changes in the global metabolic profile and to determine potential biomarkers associated with the treatment. In this case, all compounds in either the control or treatment sample can be potentially relevant. The main issue with this unbiased approach, particularly in clinical and forensic toxicology, is the relevance of compounds identified. On the other hand, the concept of biased non-targeted screening was described by Ibanez et al. [75] which involves the discovery of new compounds related to known NPS. It should be noted that in this scenario, detection involves the discovery of compounds (components) using data processing techniques and not the detection of ions by the mass spectrometer. In addition, non-targeted analysis needs to operate under the assumption that a novel analogue will be extracted using currently employed sample preparation techniques and is ionisable under routine instrumental analysis. Furthermore, the strategies presented herein are mostly for the detection of parent molecules in chemical or biological samples, however, it is possible for 
these strategies to be extended to the detection metabolites of analogues which scarcely exist as the parent molecule in biological samples (e.g. synthetic cannabinoids). It should also be considered that the detection of analogues which are often present at low or sub-ng/mL levels in biological fluids, such as synthetic cannabinoids and synthetic opioids, may be limited by HRMS sensitivity.

Generally, biased non-targeted screening is a two-step process that involves the discovery or detection of a component followed by putative identification. Component discovery has been identified as the most problematic step which can be categorized into two different approaches, top-down or bottom-up (Figure 2).

\section{Top-down non-targeted screening}

A top-down approach is best described as the most commonly employed non-targeted approach involving interrogation of mass spectral data by the selection of abundant peaks from the visual inspection of a total ion current (TIC) chromatogram. After the selection of a peak, the molecular formula can be generated from the accurate mass precursor ion along with other structural information such as (DBE).

Kneisel et al. [77] analyzed different herbal product mixtures by GC-MS and found an abundant peak that could not be identified by the current database at the time, however, it was observed that this compound had product ions similar to those formed by JWH-250. It was postulated that the compound was an $\alpha$-methyl derivative of JWH-250 (a phenylacetylindole SC) that had a molecular ion 14 mass units higher than JWH-250. Both the $\alpha$-methyl derivative and suspect compound had the same fragments under EI-MS, however, when treated with derivatising agents the respective methyl and silyl derivatives were not formed

indicating a lack of the 2-methoxyphenacetyl moiety. LC-QTOF-MS analyses showed that the product ion at $m / z \quad 135.1168$ corresponded to $\left[\mathrm{C}_{10} \mathrm{H}_{15}\right]^{+}$compared to the $\alpha$ - 
methylphenacetyl fragment $\left(\mathrm{m} / \mathrm{z}, 135.0810,\left[\mathrm{C}_{9} \mathrm{H}_{15} \mathrm{O}\right]^{+}\right)$. This product ion corresponded to an adamantoyl moiety which was determined with the assistance of NMR studies and the compound was identified as 3-(1-adamantoyl)-1-pentylindole, now known as AB-001.

An obvious limitation to top-down screening using the visual inspection of TICs is that it is only appropriate for samples that produce simple TICs with minimal abundant peaks that can be quickly located. Therefore, this technique is not well-suited to toxicological samples with complex TICs containing compounds of interest not visually obvious and abundant, potentially overlapping with endogenous components and background ions. Furthermore, the main limitation of a top-down approach is assessing the relevance of candidate peaks with respect to the compound class of interest. For instance, does the generated molecular formula, DBE and MS/MS data correlate with the compound class of interest? As a result, this approach can be a time-intensive and laborious process when multiple candidate peaks are required for interrogation.

The use of an innovative data filtering approach has been reported in an attempt to improve the efficacy of top-down approaches. Grabenauer et al. [78] reported the use of mass defect filtering (MDF) for the analysis of SCs in herbal products (Figure 3) and was able to detect JWH-250 that was not visible in the TIC. MDF, a technique commonly used in metabolomics, filters out mass spectral data based on a defined mass defect window and is effective when a class of related compounds have narrow mass defect ranges [79]. In this case, MDF was applied to samples with simple TICs, however, to our knowledge it has not been applied in an analytical toxicological context to assess its efficacy on complex sample TICs. Furthermore, it is likely that many candidate peaks are endogenous components so it is crucial that potentially exogenous peaks are prioritized for interrogation to prevent the interrogation of "false-positive" components. The removal of endogenous or background peaks can be achieved by the use of data processing algorithms to subtract TIC data of a 
representative pooled matrix or solvent blank from an authentic sample $[\underline{80}, \underline{81}]$. However, it may be difficult to obtain such a representative pooled sample that accounts for variations in endogenous component profiles in the population. A more innovative approach to identify exogenous components was reported by Cawley et al. [82], who described the use of differential analysis software for the detection of phenethylamine-type NPS in equine urine using SIEVE® (Thermo Fisher Scientific) for doping control (Figure 4). The premise of this technique is that it involves the binary comparison of $\mathrm{m} / \mathrm{z}$ data from representative matrix blanks and authentic samples to determine exogenous components and is a technique used mostly in metabolomics. Finally, most vendors provide data-mining software such as Molecular Feature Extraction (MFE, Agilent Technologies), Find by Formula (FbF, Agilent Technologies), Chromalynx XS (Waters Corporation), Trace Finder (Thermo Fisher Scientific) and Peak View (Sciex). However, the use of software-assisted peak detection approaches have typically been limited to suspect screening [ $\underline{38}, \underline{47}, \underline{63}, \underline{64}, \underline{73}, \underline{83-85]}$.

\section{Bottom-up non-targeted screening}

A bottom-up approach is an alternative technique involving the interrogation of acquired data using characteristic class-specific mass spectral information such as common product ions and neutral losses. Since this technique uses class-specific information it reduces the likelihood that "false-positive" components will be interrogated. Grabenaurer et al. [78] introduced the concept of precursor ion searching, explaining that structurally related compounds can have the same product ions and that novel analogues could be detected by searching for the precursor ions of common product ions. Recently, Pasin et al. [6] reported the characterization of hallucinogenic phenethylamines using HRMS for non-targeted screening, identifying that common product ions and neutral losses could be monitored using basic data processing techniques such as product ion searching and neutral loss filtering (NLF). Figure 5 illustrates the use of NLF with precursor neutral loss chromatograms 
(pNLC). Over the years, there has been considerable analysis of NPS analogues using HRMS, however, only some have reported the CID pathways [2-24, $\underline{46}, \underline{71}, \underline{84-88]}$. Furthermore, metabolism studies generally offer information on the CID pathways of NPS analogues since it is a conventional practice to determine the CID pathways of the parent molecule in order to identify the locations of metabolic transformations [표, $\underline{89-97]}$. As mentioned previously, the efficacy of bottom-up approaches largely relies on the acquisition of MS/MS and it has been suggested that DIA techniques should be used rather than DDA [86]. The major limitation of this approach is that it requires data processing software capable of generating numerous EICs for characteristic product ions, if monitoring all potential NPS classes. Additionally, it is possible in the future that novel analogues could potentially have different product ions due to innovative structural modifications. In this case, the use of NLFs may account for novel analogues that have unknown product ions but exhibit known neutral losses. However, NLF is a technique that is not widely incorporated into standard data processing software and has inconsistent functionality from vendor-to-vendor.

\section{Component identification}

Once a component of interest has been selected, a putative structure can be postulated from the molecular formula, DBE and interpretation of MS/MS spectra using known CID pathways $[\underline{82}, \underline{93}, \underline{95}]$. However, the assessment of MS/MS spectra requires experienced analysts who are familiar with the CID pathways of NPS. Furthermore, in silico fragmentation can be performed to assess whether the postulated structure's experimental and theoretical fragmentation correlate. This has been performed using software such as Mass Frontier $^{\mathrm{TM}}$ (Thermo Fisher Scientific) [82, 98] and Molecular Structure Correlator (MSC, Agilent Technologies) [47].

\section{Conclusions and perspectives}


The use of HRMS in forensic drug chemistry and analytical toxicology has gained popularity over recent years, providing a highly versatile analytical platform for targeted, suspect and non-targeted screening. It is evident that HRMS will ultimately become the gold-standard for the analysis of samples suspected of containing NPS due to the ability to acquire full scan MS and MS/MS that can then be retrospectively interrogated. Future developments in HRMS sensitivity will also allow it to be applied to samples containing analogues which are typically present at low concentrations in biological fluids such as synthetic cannabinoids and synthetic opioids. Presently, there is no single data processing technique that can be adopted for the reliable detection of novel analogues in non-targeted screening, however, the combination of reported techniques can be effective for the discovery of novel analogues. These reported techniques include the activity-based untargeted screening approaches such as that developed by Cannaert et al. [99] for screening synthetic cannabinoids which have the capacity to bind to cannabinoid receptors. It is envisaged that non-targeted screening becomes a highthroughput and routinely employed technique. At this stage, due to the current capabilities of vendor software, the use of this technique should be intelligence-led and reserved for samples suspected of containing NPS. Accordingly, non-targeted screening approaches will need to be updated to accommodate the advances in data processing software. The development of software-dependent top-down workflows requires analysts that are familiar and competent in the use of specific software packages. Furthermore, these workflows typically cannot be translated to other software platforms due to the exclusive nature of data processing software. Currently, bottom-up workflows offer a globally compatible and software-agnostic option since the generation of EICs for common product ions does not require specialized software. However, this approach requires the knowledge of common product ions for NPS classes that will need to be updated to include common product ions for novel derivatives. This approach can provide potential intelligence on NPS misuse on local and international levels. 


\section{Compliance with ethical standards}

Conflict of interest The authors declare that they have no conflict of interest

\section{References}

1. Collins M. Some new psychoactive substances: precursor chemicals and synthesisdriven end-products. Drug Test Anal. 2011;3:404-16.

2. Gibbons S. 'Legal Highs' - novel and emerging psychoactive drugs: a chemical overview for the toxicologist. Clin Toxicol. 2012;50:15-24.

3. Hill SL, Thomas SHL. Clinical toxicology of newer recreational drugs. Clin Toxicol. 2011;49:705-19.

4. Jerrard DA. "Designer drugs" - a current perspective. J Emerg Med. 1990;8:733-41.

5. Zawilska JB. "Legal highs" - new players in the old drama. Curr Drug Abuse Rev. 2011;4:122-30.

6. Meyer MR. New psychoactive substances: an overview on recent publications on their toxicodynamics and toxicokinetics. Arch Toxicol. 2016;90:2421-44.

7. European Drug Report 2016. Accessed in October 2016. http://www.emcdda.europa.eu/edr2016

8. $\quad$ European Drug Report 2013. Accessed in November 2016. http://www.emcdda.europa.eu/publications/edr/trends-developments/2013

9. Høiseth G, Tuv SS, Karinen R. Blood concentrations of new designer benzodiazepines in forensic cases. Forensic Sci Int. 2016;268:35-8.

10. Suzuki J, El-Haddad S. A review: Fentanyl and non-pharmaceutical fentanyls. Drug Alcohol Depend. 2017;171:107-16.

11. Archer RP, Treble R, Williams K. Reference materials for new psychoactive substances. Drug Test Anal. 2011;3:505-14. 
12. Remane D, Wissenbach DK, Peters FT. Recent advances of liquid chromatography(tandem) mass spectrometry in clinical and forensic toxicology - an update. Clin Biochem. 2016;49:1051-71.

13. Meyer MR, Du P, Schuster F, Maurer HH. Studies on the metabolism of the alphapyrrolidinophenone designer drug methylenedioxy-pyrovalerone (MDPV) in rat and human urine and human liver microsomes using GC-MS and LC-high-resolution MS and its detectability in urine by GC-MS. J Mass Spectrom. 2010;45:1426-42.

14. Meyer MR, Maurer HH. Current applications of high-resolution mass spectrometry in drug metabolism studies. Anal Bioanal Chem. 2012;403:1221-31.

15. Meyer MR, Maurer HH. Review: LC coupled to low- and high-resolution mass spectrometry for new psychoactive substance screening in biological matrices Where do we stand today? Anal Chim Acta. 2016;927:13-20.

16. Ojanpera I, Kolmonen M, Pelander A. Current use of high-resolution mass spectrometry in drug screening relevant to clinical and forensic toxicology and doping control. Anal Bioanal Chem. 2012;403:1203-20.

17. Maurer HH, Meyer MR. High-resolution mass spectrometry in toxicology: current status and future perspectives. Arch Toxicol. 2016;90:2161-72.

18. Scientific Working Group for the Analysis of Seized Drugs Recommendations. Accessed in December 2016. http://www.swgdrug.org/approved

19. Wu AH, Gerona R, Armenian P, French D, Petrie M, Lynch KL. Role of liquid chromatography-high-resolution mass spectrometry (LC-HR/MS) in clinical toxicology. Clin Toxicol. 2012;50:733-42.

20. Peters FT. Recent developments in urinalysis of metabolites of new psychoactive substances using LC-MS. Bioanalysis. 2014;6:2083-107. 
21. Ellefsen KN, Concheiro M, Huestis MA. Synthetic cathinone pharmacokinetics, analytical methods, and toxicological findings from human performance and postmortem cases. Drug metabolism reviews. 2016:1-29.

22. Shevyrin V, Melkozerov V, Eltsov O, Shafran Y, Morzherin Y. Synthetic cannabinoid 3-benzyl-5-[1-(2-pyrrolidin-1-ylethyl)-1H-indol-3-yl]-1,2,4-oxadiazole. The first detection in illicit market of new psychoactive substances. Forensic Sci Int. 2016;259:95-100.

23. Shevyrin V, Melkozerov V, Nevero A, Eltsov O, Morzherin Y, Shafran Y. 3Naphthoylindazoles and 2-naphthoylbenzoimidazoles as novel chemical groups of synthetic cannabinoids: chemical structure elucidation, analytical characteristics and identification of the first representatives in smoke mixtures. Forensic Sci Int. $2014 ; 242: 72-80$.

24. Shevyrin V, Melkozerov V, Nevero A, Eltsov O, Shafran Y, Morzherin Y, et al. Identification and analytical characteristics of synthetic cannabinoids with an indazole-3-carboxamide structure bearing a N-1-methoxycarbonylalkyl group. Anal Bioanal Chem. 2015;407:6301-15.

25. Sekuła K, Zuba D. Structural elucidation and identification of a new derivative of phenethylamine using quadrupole time-of-flight mass spectrometry. Rapid Commun Mass Spectrom. 2013;27:2081-90.

26. Zuba D, Sekula K. Analytical characterization of three hallucinogenic N-(2methoxy)benzyl derivatives of the $2 \mathrm{C}$-series of phenethylamine drugs. Drug Test Anal. 2013;5:634-45.

27. Zuba D, Sekuła K, Buczek A. 25C-NBOMe - New potent hallucinogenic substance identified on the drug market. Forensic Sci Int. 2013;227:7-14. 
28. Glicksberg L, Bryand K, Kerrigan S. Identification and quantification of synthetic cathinones in blood and urine using liquid chromatography-quadrupole/time of flight (LC-Q/TOF) mass spectrometry. J Chromatogr B. 2016;1035:91-103.

29. Soh YN, Elliott S. An investigation of the stability of emerging new psychoactive substances. Drug Test Anal. 2014;6:696-704.

30. Montesano C, Vannutelli G, Gregori A, Ripani L, Compagnone D, Curini R, et al. Broad screening andiIdentification of novel psychoactive substances in plasma by high-performance liquid chromatography-high-resolution mass spectrometry and post-run library matching. J Anal Toxicol. 2016;40:519-28.

31. Pasin D, Bidny S, Fu S. Analysis of new designer drugs in post-mortem blood using high-resolution mass spectrometry. J Anal Toxicol. 2015;39:163-71.

32. Wozniakiewicz A, Wietecha-Posluszny R, Wozniakiewicz M, Bryczek E, Koscielniak P. A quick method for determination of psychoactive agents in serum and hair by using capillary electrophoresis and mass spectrometry. J Pharm Biomed Anal. 2015;111:177-85.

33. Concheiro M, Anizan S, Ellefsen K, Huestis MA. Simultaneous quantification of 28 synthetic cathinones and metabolites in urine by liquid chromatography-high resolution mass spectrometry. Anal Bioanal Chem. 2013;405:9437-48.

34. Concheiro M, Castaneto M, Kronstrand R, Huestis MA. Simultaneous determination of 40 novel psychoactive stimulants in urine by liquid chromatography-high resolution mass spectrometry and library matching. J Chromatogr A. 2015;1397:3242.

35. Archer JR, Dargan PI, Hudson S, Wood DM. Analysis of anonymous pooled urine from portable urinals in central London confirms the significant use of novel psychoactive substances. QJM. 2013;106:147-52. 
36. Archer JR, Hudson S, Jackson O, Yamamoto T, Lovett C, Lee HM, et al. Analysis of anonymized pooled urine in nine UK cities: variation in classical recreational drug, novel psychoactive substance and anabolic steroid use. QJM. 2015;108:929-33.

37. Archer JR, Dargan PI, Lee HM, Hudson S, Wood DM. Trend analysis of anonymised pooled urine from portable street urinals in central London identifies variation in the use of novel psychoactive substances. Clin Toxicol. 2014;52:160-5.

38. Andres-Costa MJ, Andreu V, Pico Y. Analysis of psychoactive substances in water by information dependent acquisition on a hybrid quadrupole time-of-flight mass spectrometer. J Chromatogr A. 2016;1461:98-106.

39. Baz-Lomba JA, Reid MJ, Thomas KV. Target and suspect screening of psychoactive substances in sewage-based samples by UHPLC-QTOF. Anal Chim Acta. 2016;914:81-90.

40. Gonzalez-Marino I, Gracia-Lor E, Bagnati R, Martins CP, Zuccato E, Castiglioni S. Screening new psychoactive substances in urban wastewater using high resolution mass spectrometry. Anal Bioanal Chem. 2016;408:4297-309.

41. Backberg M, Tworek L, Beck O, Helander A. Analytically Confirmed Intoxications Involving MDMB-CHMICA from the STRIDA Project. J Med Toxicol. 2017;13:5260.

42. Sundstrom M, Pelander A, Simojoki K, Ojanpera I. Patterns of drug abuse among drug users with regular and irregular attendance for treatment as detected by comprehensive UHPLC-HR-TOF-MS. Drug Test Anal. 2016;8:39-45.

43. Sundstrom M, Pelander A, Angerer V, Hutter M, Kneisel S, Ojanpera I. A highsensitivity ultra-high performance liquid chromatography/high-resolution time-offlight mass spectrometry (UHPLC-HR-TOFMS) method for screening synthetic 
cannabinoids and other drugs of abuse in urine. Anal Bioanal Chem. 2013;405:846374.

44. Frison G, Frasson S, Zancanaro F, Tedeschi G, Zamengo L. Detection of 3methylmethcathinone and its metabolites 3-methylephedrine and 3methylnorephedrine in pubic hair samples by liquid chromatography-high resolution/high accuracy Orbitrap mass spectrometry. Forensic Sci Int. 2016;265:1317.

45. Montesano C, Vannutelli G, Massa M, Simeoni MC, Gregori A, Ripani L, et al. Multi-class analysis of new psychoactive substances and metabolites in hair by pressurized liquid extraction coupled to HPLC-HRMS. Drug Test Anal. 2016. doi:10.1002/dta.2043

46. Fornal E. Identification of substituted cathinones: 3,4-Methylenedioxy derivatives by high performance liquid chromatography-quadrupole time of flight mass spectrometry. J Pharm Biomed Anal. 2013;81-82:13-9.

47. Paul M, Ippisch J, Herrmann C, Guber S, Schultis W. Analysis of new designer drugs and common drugs of abuse in urine by a combined targeted and untargeted LC-HRQTOFMS approach. Anal Bioanal Chem. 2014;406:4425-41.

48. Zaitsu K, Nakayama H, Yamanaka M, Hisatsune K, Taki K, Asano T, et al. Highresolution mass spectrometric determination of the synthetic cannabinoids MAM2201, AM-2201, AM-2232, and their metabolites in postmortem plasma and urine by LC/Q-TOFMS. Int J Legal Med. 2015;129:1233-45.

49. Ojanpera I, Mesihaa S, Rasanen I, Pelander A, Ketola RA. Simultaneous identification and quantification of new psychoactive substances in blood by GCAPCI-QTOFMS coupled to nitrogen chemiluminescence detection without authentic reference standards. Anal Bioanal Chem. 2016;408:3395-400. 
50. Baciu T, Botello I, Borrull F, Calull M, Aguilar C. Capillary electrophoresis and related techniques in the determination of drugs of abuse and their metabolites. TrAC, Trends Anal Chem. 2015;74:89-108.

51. Alechaga E, Moyano E, Galceran MT. Wide-range screening of psychoactive substances by FIA-HRMS: identification strategies. Anal Bioanal Chem. 2015;407:4567-80.

52. Ostermann KM, Luf A, Lutsch NM, Dieplinger R, Mechtler TP, Metz TF, et al. MALDI Orbitrap mass spectrometry for fast and simplified analysis of novel street and designer drugs. Clin Chem Acta. 2014;433:254-8.

53. Stojanovska N, Kelly T, Tahtouh M, Beavis A, Fu S. Analysis of amphetamine-type substances and piperazine analogues using desorption electrospray ionisation mass spectrometry. Rapid Commun Mass Spectrom. 2014;28:731-40.

54. Stojanovska N, Tahtouh M, Kelly T, Beavis A, Fu S. Presumptive analysis of 4methylmethcathinone (mephedrone) using desorption electrospray ionisation - mass spectrometry (DESI-MS). Aust J Forensic Sci. 2014;46:411-23.

55. Botch-Jones S, Foss J, Barajas D, Kero F, Young C, Weisenseel J. The detection of NBOMe designer drugs on blotter paper by high resolution time-of-flight mass spectrometry (TOFMS) with and without chromatography. Forensic Sci Int. 2016;267:89-95.

56. Acton WJ, Lanza M, Agarwal B, Jurschik S, Sulzer P, Breiev K, et al. Headspace analysis of new psychoactive substances using a selective reagent ionisation-time of flight-mass spectrometer. Int J Mass Spectrom. 2014;360:28-38.

57. Lanza M, Acton WJ, Sulzer P, Breiev K, Jurschik S, Jordan A, et al. Selective reagent ionisation-time of flight-mass spectrometry: a rapid technology for the novel analysis of blends of new psychoactive substances. J Mass Spectrom. 2015;50:427-31. 
58. Habala L, Valentova J, Pechova I, Fuknova M, Devinsky F. DART - LTQ

ORBITRAP as an expedient tool for the identification of synthetic cannabinoids. Leg Med. 2016;20:27-31.

59. Poklis JL, Wolf CE, ElJordi OI, Liu K, Zhang S, Poklis A. Analysis of the first- and second-generation Raving Dragon Novelty Bath Salts containing methylone and pentedrone. J For Sci. 2015;60:S234-40.

60. Musah RA, Cody RB, Domin MA, Lesiak AD, Dane AJ, Shepard JR. DART-MS insource collision induced dissociation and high mass accuracy for new psychoactive substance determinations. Forensic Sci Int. 2014;244:42-9.

61. Gwak S, Almirall JR. Rapid screening of 35 new psychoactive substances by ion mobility spectrometry (IMS) and direct analysis in real time (DART) coupled to quadrupole time-of-flight mass spectrometry (QTOF-MS). Drug Test Anal. 2015;7:884-93.

62. Qian Z, Hua Z, Liu C, Jia W. Four types of cannabimimetic indazole and indole derivatives, ADB-BINACA, AB-FUBICA, ADB-FUBICA, and AB-BICA, identified as new psychoactive substances. Forensic Toxicol. 2016;34:133-43.

63. Kinyua J, Negreira N, Ibáñez M, Bijlsma L, Hernández F, Covaci A, et al. A dataindependent acquisition workflow for qualitative screening of new psychoactive substances in biological samples. Anal Bioanal Chem. 2015;407:8773-85.

64. Kinyua J, Negreira N, Miserez B, Causanilles A, Emke E, Gremeaux L, et al. Qualitative screening of new psychoactive substances in pooled urine samples from Belgium and United Kingdom. Sci Total Environ. 2016;573:1527-35.

65. Oberacher H, Arnhard K. Current status of non-targeted liquid chromatographytandem mass spectrometry in forensic toxicology. TrAC, Trends Anal Chem. 2016;84:94-105. 
66. Zuba D, Sekuła K. Identification and characterization of 2,5-dimethoxy-3,4-dimethyl$\beta$-phenethylamine (2C-G) - A new designer drug. Drug Test Anal. 2013;5:549-59.

67. Zuba D, Sekuła K, Buczek A. Identification and characterization of 2,5-dimethoxy-4nitro- $\beta$-phenethylamine $(2 \mathrm{C}-\mathrm{N})-\mathrm{A}$ new member of $2 \mathrm{C}$-series of designer drug. Forensic Sci Int. 2012;222:298-305.

68. Schymanski EL, Singer HP, Slobodnik J, Ipolyi IM, Oswald P, Krauss M, et al. Nontarget screening with high-resolution mass spectrometry: critical review using a collaborative trial on water analysis. Anal Bioanal Chem. 2015;407:6237-55.

69. Krauss M, Singer H, Hollender J. LC-high resolution MS in environmental analysis: from target screening to the identification of unknowns. Anal Bioanal Chem. 2010;397:943-51.

70. Heikman P, Sundstrom M, Pelander A, Ojanpera I. New psychoactive substances as part of polydrug abuse within opioid maintenance treatment revealed by comprehensive high-resolution mass spectrometric urine drug screening. Hum Psychopharmacol. 2016;31:44-52.

71. Langer N, Lindigkeit R, Schiebel H-M, Papke U, Ernst L, Beuerle T. Identification and quantification of synthetic cannabinoids in "spice-like" herbal mixtures: Update of the German situation for the spring of 2016. Forensic Sci Int. 2016;269:31-41.

72. Lung D, Wilson N, Chatenet FT, LaCroix C, Gerona R. Non-targeted screening for novel psychoactive substances among agitated emergency department patients. Clin Toxicol. 2016;54:319-23.

73. Ford LT, Berg JD. Analysis of legal high materials by UPLC-MS/TOF as part of a toxicology vigilance system. What are the most popular novel psychoactive substances in the UK? Ann Clin Biochem. 2016;54:219-29. 
74. Reid MJ, Baz-Lomba JA, Ryu Y, Thomas KV. Using biomarkers in wastewater to monitor community drug use: a conceptual approach for dealing with new psychoactive substances. Sci Total Environ. 2014;487:651-8.

75. Ibáñez M, Sancho JV, Bijlsma L, van Nuijs ALN, Covaci A, Hernández F. Comprehensive analytical strategies based on high-resolution time-of-flight mass spectrometry to identify new psychoactive substances. TrAC, Trends Anal Chem. 2014;57:107-17.

76. Knolhoff AM, Croley TR. Non-targeted screening approaches for contaminants and adulterants in food using liquid chromatography hyphenated to high resolution mass spectrometry. J Chromatogr A. 2016;1428:86-96.

77. Kneisel S, Westphal F, Bisel P, Brecht V, Broecker S, Auwarter V. Identification and structural characterization of the synthetic cannabinoid 3-(1-adamantoyl)-1pentylindole as an additive in 'herbal incense'. J Mass Spectrom. 2012;47:195-200.

78. Grabenauer M, Krol WL, Wiley JL, Thomas BF. Analysis of synthetic cannabinoids using high-resolution mass spectrometry and mass defect filtering: implications for nontargeted screening of designer drugs. Anal Chem. 2012;84:5574-81.

79. Sleno L. The use of mass defect in modern mass spectrometry. J Mass Spectrom. 2012;47:226-36.

80. Broecker S, Herre S, Wüst B, Zweigenbaum J, Pragst F. Development and practical application of a library of CID accurate mass spectra of more than 2,500 toxic compounds for systematic toxicological analysis by LC-QTOF-MS with datadependent acquisition. Anal Bioanal Chem. 2011;400:101-17.

81. Broecker S, Pragst F, Bakdash A, Herre S, Tsokos M. Combined use of liquid chromatography-hybrid quadrupole time-of-flight mass spectrometry (LC-QTOFMS) and high performance liquid chromatography with photodiode array detector 
(HPLC-DAD) in systematic toxicological analysis. Forensic Sci Int. 2011;212:21526.

82. Cawley A, Pasin D, Ganbat N, Ennis L, Smart C, Greer C, et al. The potential for complementary targeted/non-targeted screening of novel psychoactive substances in equine urine using liquid chromatography-high resolution accurate mass spectrometry. Anal Methods. 2016;8:1789-97.

83. Ibanez M, Bijlsma L, van Nuijs AL, Sancho JV, Haro G, Covaci A, et al. Quadrupoletime-of-flight mass spectrometry screening for synthetic cannabinoids in herbal blends. J Mass Spectrom. 2013;48:685-94.

84. Fornal E. Formation of odd-electron product ions in collision-induced fragmentation of electrospray-generated protonated cathinone derivatives: aryl $\alpha$-primary amino ketones. Rapid Commun Mass Spectrom. 2013;27:1858-66.

85. Fornal E. Study of collision-induced dissociation of electrospray-generated protonated cathinones. Drug Test Anal. 2014;6:705-15.

86. Pasin D, Cawley A, Bidny S, Fu S. Characterisation of hallucinogenic phenethylamines using high-resolution mass spectrometry for non-targeted screening purposes. Drug Test Anal. 2017. doi:10.1002/dta.2171

87. Fornal E, Stachniuk A, Wojtyla A. LC-Q/TOF mass spectrometry data driven identification and spectroscopic characterisation of a new 3,4-methylenedioxy-Nbenzyl cathinone (BMDP). J Pharm Biomed Anal. 2013;72:139-44.

88. Strano Rossi S, Odoardi S, Gregori A, Peluso G, Ripani L, Ortar G, et al. An analytical approach to the forensic identification of different classes of new psychoactive substances (NPSs) in seized materials. Rapid Commun Mass Spectrom. 2014;28:1904-16. 
89. Andersson M, Diao X, Wohlfarth A, Scheidweiler KB, Huestis MA. Metabolic profiling of new synthetic cannabinoids AMB and 5F-AMB by human hepatocyte and liver microsome incubations and high-resolution mass spectrometry. Rapid Commun Mass Spectrom. 2016;30:1067-78.

90. Diao X, Wohlfarth A, Pang S, Scheidweiler KB, Huestis MA. High-resolution mass spectrometry for characterizing the metabolism of synthetic cannabinoid THJ-018 and its 5-fluoro analog THJ-2201 after incubation in human hepatocytes. Clin Chem. 2016;62:157-69.

91. Ellefsen KN, Wohlfarth A, Swortwood MJ, Diao X, Concheiro M, Huestis MA. 4Methoxy-alpha-PVP: in silico prediction, metabolic stability, and metabolite identification by human hepatocyte incubation and high-resolution mass spectrometry. Forensic Toxicol. 2016;34:61-75.

92. Swortwood MJ, Ellefsen KN, Wohlfarth A, Diao X, Concheiro-Guisan M, Kronstrand R, et al. First metabolic profile of PV8, a novel synthetic cathinone, in human hepatocytes and urine by high-resolution mass spectrometry. Anal Bioanal Chem. 2016;408:4845-56.

93. Watanabe S, Kuzhiumparambil U, Winiarski Z, Fu S. Biotransformation of synthetic cannabinoids JWH-018, JWH-073 and AM2201 by Cunninghamella elegans. Forensic Sci Int. 2016;261:33-42.

94. Wohlfarth A, Pang S, Zhu M, Gandhi AS, Scheidweiler KB, Liu HF, et al. First metabolic profile of XLR-11, a novel synthetic cannabinoid, obtained by using human hepatocytes and high-resolution mass spectrometry. Clin Chem. 2013;59:1638-48.

95. Watanabe S, Kuzhiumparambil U, Nguyen MA, Cameron J, Fu S. Metabolic profile of synthetic cannabinoids 5F-PB-22, PB-22, XLR-11 and UR-144 by Cunninghamella elegans. AAPS J. 2017. doi:10.1208/s12248-017-0078-4 
96. Caspar AT, Helfer AG, Michely JA, Auwarter V, Brandt SD, Meyer MR, et al. Studies on the metabolism and toxicological detection of the new psychoactive designer drug 2-(4-iodo-2,5-dimethoxyphenyl)-N-[(2methoxyphenyl)methyl]ethanamine (25I-NBOMe) in human and rat urine using GCMS, LC-MS(n), and LC-HR-MS/MS. Anal Bioanal Chem. 2015;407:6697-719.

97. Franz F, Angerer V, Moosmann B, Auwärter V. Phase I metabolism of the highly potent synthetic cannabinoid MDMB-CHMICA and detection in human urine samples. Drug Test Anal. 2016. doi:10.1002/dta.2049

98. Lobo Vicente J, Chassaigne H, Holland MV, Reniero F, Kolar K, Tirendi S, et al. Systematic analytical characterization of new psychoactive substances: A case study. Forensic Sci Int. 2016;265:107-15.

99. Cannaert A, Storme J, Franz F, Auwärter V, Stove CP. Detection and Activity Profiling of Synthetic Cannabinoids and Their Metabolites with a Newly Developed Bioassay. Anal Chem. 2016;88:11476-85. 